\title{
Improved Algorithm for Image Segmentation based on the Three-dimensional Reconstruction of Tumor Images
}

\author{
Jianning Han ${ }^{1}$, Quan Zhang ${ }^{1}$, Peng Yang ${ }^{1}$ and Yifan Gong ${ }^{1}$ \\ ${ }^{1}$ School of Information and Communication Engineering, North University of \\ China, \\ Tai Yuan, Shan Xi, 030051 \\ ${ }^{1}$ Tel.: 13994203384 \\ ${ }^{1}$ E-mail: yp7458@sina.cn
}

\begin{abstract}
Recently, more and more researchers have been paying close attention to tumor image processing techniques, in which the tumor image segmentation technology is regarded as the absolute research focus. Given that there exist only one tumor image segmentation algorithm, no further high quality three-dimensional slice can be provided for the later reconstruction of the three-dimensional reconstruction of tumor images. In this paper, we propose an improved segmentation algorithm by combining the fuzzy clustering segmentation with the fuzzy edge enhancement. Finally, the experimental results show that the fuzzy clustering segmentation is highly efficient for dealing with the fuzziness and complexity of brain tissues, and the images after the fuzzy clustering segmentation calculated by the edge detection algorithm of fuzzy enhancement have more clear edges, which provides a solid foundation for further 3D processing and finally helps achieve better $3 D$ visualization effect on brain tumors. Our algorithm provides promising application prospect in the field of the medical image processing.
\end{abstract}

Keywords: Brain tumor images; Watershed algorithm; Fuzzy clustering; Fuzzy enhancement; Edge detection

\section{Introduction}

In recent years, the brain tumor incidence has been on the rise. Nowadays the diagnosis and treatment of brain tumor mainly depend on the image technology of brain tumor images. In this community, an increasing number of medical image researchers are attaching great importance to the brain tumor image processing technology, especially to the brain image segmentation [1]. However, the problems suffered by brain tumor image segmentation lies in its complexity. Specifically, tissues and organs of a human body have irregular shapes and there are also differences between individual people. Therefore, the brain tumor image segmentation is the basis of a series of further operations, such as quantitative analysis of normal tissues and the diseased ones and three-dimensional reconstruction, but at the same time it's also a bottleneck of brain tumor medical testing [2].

In the detection of the brain tumor medical image segmentation, researchers concentrate mainly on segmentations of the three-dimensional CT and the MRI image. According to algorithms, there can be three major categories, namely the segmentation method based on structure, statistics and the mix method [3]. Among them, the watershed algorithm and the edge detection algorithm are two typical ones. But the traditional algorithms for different images have a certain effect, but as these things mainly concerns the effect of a single aspect, it will bring about some problems such as details missing, edge blur in the later processing, thus influencing the later 3D reconstruction. Therefore, 
this paper tries to solve the key problem of the brain tumor medical image segmentation by combining the brain tumor image characteristics with the research that the $3 \mathrm{D}$ reconstruction needs comprehensive image segmentation algorithm [4].

\section{Limits of the Traditional Brain Tumor Image Segmentation Algorithm}

The brain tumor image segmentation is about dividing the brain tumor image into sets of different attributes, mainly to highlight the neurocranium and tumor tissue. As contourof neurocranium and tumor often refer to the area with the large grey level variation range, most researchers choose the watershed algorithm. But during the process, the superfluous local extremum, which appears when the gradient algorithm is used to deal with brain tumor image information in uniform distribution, will generate many "bottom valleys" and "catchment basins". Eventually, the contour of the original brain tumor image will be difficult to identify in a cluttered watershed (Figure 1a). In an image with "over-segmentation" (Figure 1b), one can hardly find the edge information of the brain and tumor [5].

Having realized the existence of over-segmentation, researchers found that noise could usually be found in brain tumor images. As the watershed algorithm is extremely sensitive to noise, the noise can directly affect the gradient calculation of image and cause the position offset of the outline of image segmentation. Therefore, some researches will first do the smooth processing before they use the watershed transform to reduce over-segmentation phenomenon caused by too many "catchment basins". Figure 1c shows the effect picture with watershed algorithm after the smoothness processing, in which we can find the external rim of neurocranium becomes clear, but the internal details are still unclear. This is due to the low contrast ratio of brain tumor image itself, and the gradient value of the regional boundary pixel does not change obviously. In order to improve the segmentation performance, some authors put forward the idea that we could introduce the mathematical morphology filtering to help the watershed algorithm. First of all, processing the original brain tumor image by using adaptive filtering (Figure 1a), then use top-hat and bot-hat transformation on the filtering image, and use the algebraic operation method to expand clearance and enhance the image, and finally use the watershed algorithm to process the image. Figure 1d shows the watershed algorithm result based on the mathematical morphological filtering, and obviously the internal details and external boundary of brain tumor have better results [6].

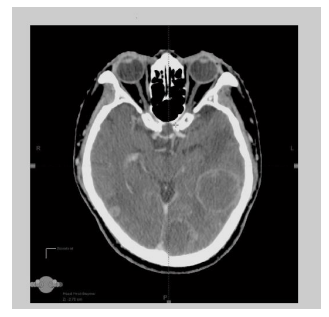

(a) The original brain tumor image

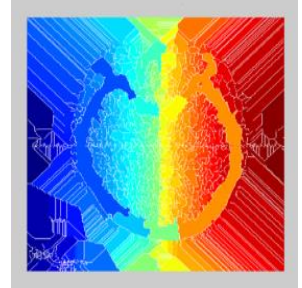

(c)The watershed segmentation result after the filtering

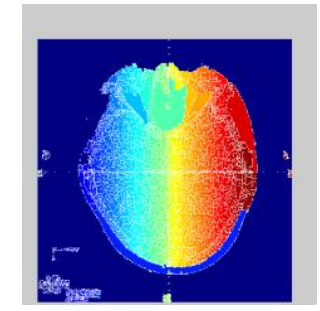

(b) The image of over-segmentation

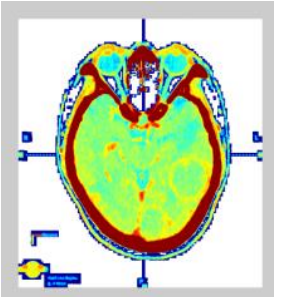

(d) The improved watershed segmentation image

Figure 1. The Traditional Segmentation Algorithm of Brain Tumor Images 
As the basis of brain tumor image 3D reconstruction, the application of the brain tumor image segmentation must consider the applicability in post processing. In order to test the improved watershed algorithm comprehensively, the images after segmentation are disposed respectively with edge detection operators (Prewitt, Roberts, Log, canny, etc.). It can be found that while the improved watershed algorithm has achieved promising image segmentation effect, the effect after the edge detection processing is far away from ideal. Firstly, the derivative operator is used to analyze the whole image and then do a threshold processing to the computing results. After calculating the edge intensity of each pixel in the image, pick up the points whose edge intensity are greater than a certain value and assign them to pixel value " 1 " and assign the rest to pixel value " 0 ". Therefore, the edge point set can be extracted from the image. However, as the internal brain tumor edge value and the surrounding gray value are very close in Figure 1d, it will make the edge point set difficult to identify. As we can see from Figure 2, details in brain tumor, especially the boundary inside the brain tumor, are all disappear and certainly it cannot provide the high quality of slice images for the post 3D reconstruction, directly affecting the medical detection and diagnosis of brain tumors. So the traditional brain tumor image segmentation algorithms cannot meet the need of the brain tumor 3D visualization and must be comprehensively improved for the post processing.

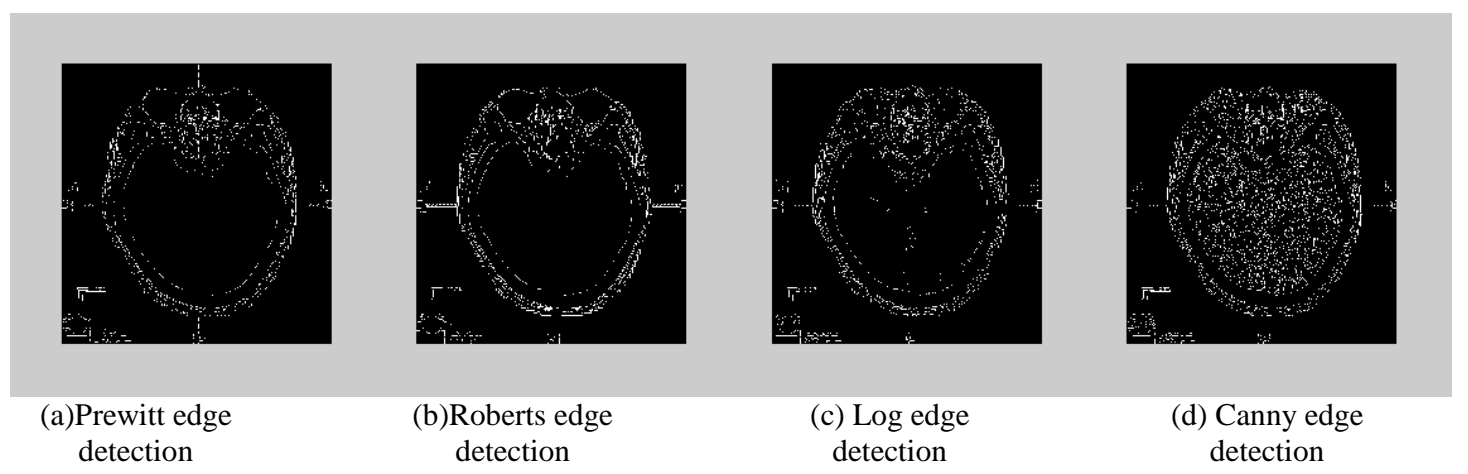

Figure 2. Edge Detection Results of Four Common Operator

\section{Comprehensive Improvement of the Brain Tumor Image Segmentation Algorithm}

There is much changeable gray level information and marginal information in the brain tumor image. So to segment the image based on only the gray level difference of the object is not enough, for the information also shows the differences in texture image or other statistics parameter derived from the image grey level. Therefore, according to the fuzzy clustering algorithm, this paper makes full use of gray level, texture and other statistical parameters of brain tumor for clustering analysis, and chooses the appropriate feature variables. The identified object point (brain tumor, and other normal tissues of the brain) will distribute in the form of clusters in a multidimensional feature space. And at the same time, the edge details of brain image in each tissue (especially the brain tumor) can be well preserved, which is very useful. Therefore, this paper puts forward the idea that we should use the comprehensive improvement algorithm based on the fuzzy $\mathrm{C}$-average clustering and the fuzzy enhancement edge detection in brain image segmentation [7].

The Fuzzy Clustering Method has made the C-average clustering algorithm extend to the fuzzy field. When using FCM algorithm for calculation, firstly, we need to define the objective function and minimize it. Usually the objective function definition is:

$$
J(U, V)=\sum_{k=1}^{n} \sum_{i=1}^{c} u_{i k}^{m}\left\|x_{i}-v_{i}\right\|^{2}=\sum_{k=1}^{n} \sum_{i=1}^{c} u_{i k}^{m}\left(d_{i k}\right)^{2}
$$


$x_{k}$ and $v_{i}$ represent the data points and the center point in the formula, $d_{i k}$ is the distance between them, $m \in(1, \infty)$ is the weighting coefficient, and when the $m=1$, it is C - average algorithm. To solve the conditional extremum $(U, V)$ of the objective function $J(U, V)$, we use the Lagrange algorithm:

$$
u_{i k}=\frac{v_{i}=\frac{\sum_{k=1}^{n} u_{i k}^{m} x_{k}}{\sum_{k=1}^{n} u_{i k}^{m}} \quad(\mathrm{i}=1,2, \cdots, \mathrm{c})}{\sum_{j=1}^{c}\left\{\frac{d_{i k}}{d_{j k}}\right\}^{2 /(m-1)}} \quad(\mathrm{i}=1,2, \cdots, \mathrm{c} ; \mathrm{k}=1,2, \cdots, \mathrm{n})
$$

According to the core of FCM algorithm, firstly we need to use the similarity and distance measure as the criteria of clustering analysis to define similarity measure function (Euclidean distance), and conduct feature extraction and selection according to the clustering objects. Use the clustering validity function to make the cluster computing and result evaluation. Due to the complexity of the brain tumor image and considering that the clustering will affect object choice and similarity measure, our algorithm specifically adds the feedback control for the best effect of clustering analysis [8].

It is known that the brain tumor clustering image provides the high quality image for the post image edge detection. However, if using the traditional edge detection algorithm, we can make the derivative operator which is sensitive to the pixel grayscale step change within the airspace. Because the derivative operator has a numerical value in grayscale, this kind of derivative operator (such as gradient, Laplace operator, etc.) can be used as the edge detector. First we can use these derivative operators calculate the whole image, and then make a threshold processing for the computing results so that the edge point set can be extracted from the image. But when common edge detection operators (Prewitt, Roberts, Log, canny, etc.) is used to process an image for a brain tumor, it is based on a template and the convolution with the corresponding area and the result can be used as the edge strength of central part of this area in the image. After that, value points whose edge strength are greater than some constant value can be extracted, and assign them to pixel value of " 1 ", the rest of them assign pixel value " 0 ". If so, the testing of the internal image edges can be unsystematic, the lines cannot be connected, and we can't see the internal configuration and structure of the image clearly. Therefore, combining the fuzzy mathematics thought above, we propose to use this edge detection method of fuzzy enhancement after clustering the images. First of all, according to the OSTU algorithm, we calculate the appropriate threshold, define a new membership function, use the fuzzy enhancement algorithm for edge detection, adjust parameter values based on the characteristics of the image, and then use the "min" operator of Nakagowa and Rosenfeld to extract the image edge. After this process, we can overcome the defects that many original images with low grey value by cutting to zero after the transformation of the ordinary operator testing. Moreover, defining the membership degree in a low gray level region and high gray level region respectively in the image also guarantees that the image information loss in the low gray level area remains less obvious, making the following fuzzy enhancement achieve good results.

Based on the analysis above, the comprehensive improvement algorithm of fuzzy $\mathrm{C}$-average clustering with fuzzy enhance edge detection specific algorithm process is illustrated as follows: 


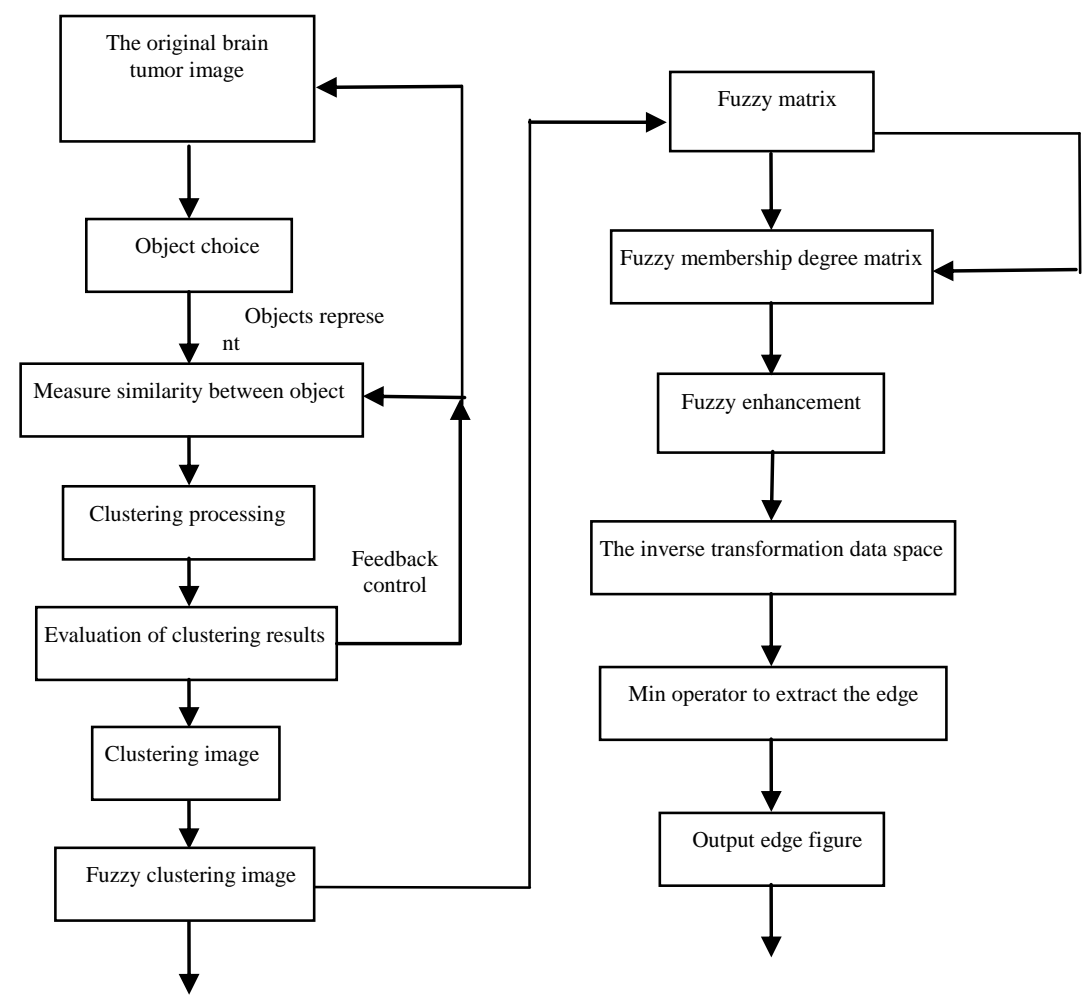

Figure 3. Image Segmentation Algorithm Flowchart Comprehensive Improvement

\section{The Steps and Implementation of the Comprehensive Improvement Algorithm for the Brain Tumor Image Segmentation}

4.1. The Steps and Implementation of Fuzzy Clustering Segmentation of the Brain Tumor Image

(1) Determine the type of clustering number " $C$ ", $2 \leq C \leq n$; Determine the weighted index"m”, $1 \leq m \leq \infty$, stop the iteration conditions $\varepsilon$, initially divide matrix $U^{(0)}$, $V^{(0)}$ (assuming each step with $\mathrm{r}$ tags, $\left.\mathrm{r}=0,1, \ldots\right)$;

(2) According to Formulation (2), calculate each step C center $\left\{V_{i}^{(r)}\right\}$; according to Formulation (3), modify each step of membership degree matrix $U^{(r)}$, when $d_{i k}=\left\|x_{k}-v_{i}\right\| \neq 0$,

$$
u_{i k}^{(r+1)}=\left[\sum_{j=1}^{C}\left\{\frac{d_{i k}^{(r)}}{d_{j k}^{(r)}}\right\}^{2 /(m-1)}\right]^{1} ;
$$

when $d_{i k}=\left\|x_{k}-v_{i}\right\|=0$, suppose $I_{k}=\left\{i \mid 2 \leq c \leq n ; d_{i k}^{(r)}=0\right\}, \quad \overline{I_{k}}=\{1,2, \cdot c\}-I_{k}$, for all $\mathrm{i}, i \in \overline{I_{k}}$, Meet the $u_{i k}^{(r+1)}=0, \sum_{i \in I_{k}} u_{i k}^{(r+1)}=1$.

(3) If $\left\|U^{(r+1)}-U^{(r)}\right\| \leq \varepsilon$, stop the iteration, otherwise $r=r+1$ and return step (2)

(4) Process clustering image segmentation after suitable brain tumor images are created. 
According to the steps above, run the program under the MATLAB and get the corresponding results as, Figure 4 (a) is a primitive brain tumor image and Figure 4 (b) is the segmentation result after fuzzy clustering. By comparing the improved watershed algorithm from Figure 4 (b) with Figure 1 (d), we can see fuzzy C-average for image segmentation effect is better. It can not only achieve a good effect of the traditional watershed algorithm, but also efficiently deal with brain tumor edge information. The method is very suitable for brain tumor images with fuzziness and uncertainty.

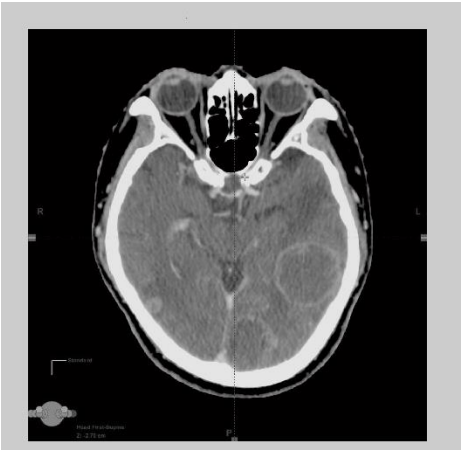

(a)The original brain image

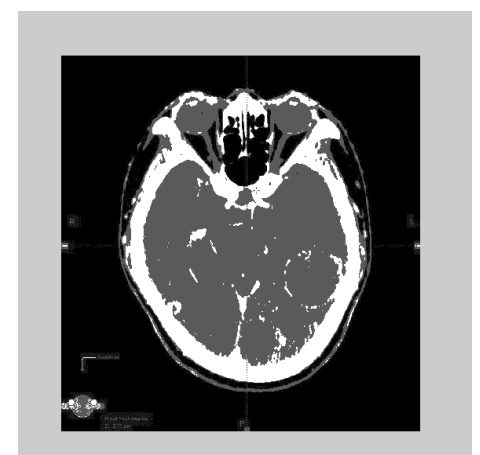

(b) The result after fuzzy clustering segmentation

Figure 4. The Image Comparison after Fuzzy Clustering Segmentation

\subsection{The Steps and Implementation of the Fuzzy Enhancement Edge Detection Algorithm for the Brain Tumor Fuzzy Clustering Image}

(1) According to the characteristics of the fuzzy clustering of the brain tumor image, rely on the OSTU algorithm to calculate threshold XT; calculate the maximum variance and get the optimal threshold value.

(2) Based on the characteristics of the brain tumor image, adjust the value of $\mu_{c}$, choose the most appropriate $\mu_{c} \quad\left(0<\mu_{c}<1\right)$, and then define a new membership function:

$$
p_{i j}=T\left(x_{i j}\right)=\left\{\begin{array}{cc}
x_{i j} / X_{T} & x_{i j} \leq X_{T} \\
x_{i j} / X_{\max } & x_{i j}>X_{T}
\end{array}\right.
$$

$\mathrm{x}_{\mathrm{ij}}$ is the the original data matrix of input image, $\mathrm{XT}$ is the threshold.

(3) Repeatedly use the type (5) of the enhancement of the operator, make fuzzy enhancement of the brain tumor image. Such transformation increase $p_{i j}$ that is more than the value $\mu_{c}$ and reduces $p_{i j}$ that is less than value $\mu_{c}$. The $\mu_{c}$ here has evolved into a generalized transition point:

$$
\begin{array}{r}
p_{i j}{ }^{\prime}=I_{r}\left(p_{i j}\right)=I_{1}\left(I_{r-1}\left(p_{i j}\right)\right), r=1,2,3, \cdots \\
I_{1}\left(p_{i j}\right)=\left\{\begin{array}{cc}
p_{i j}{ }^{2} / u_{c} & 0 \leq p_{i j} \leq u_{c} \\
1-\left(1-p_{i j}\right)^{2} /\left(1-u_{c}\right) & u_{c} \leq p_{i j} \leq 1
\end{array}\right.
\end{array}
$$

(4) According to the type (6), conduct the $\mathrm{T}-1$ transform for the brain tumor image. Transform the brain images of fuzzy enhancement from fuzzy domain to spatial domain.

$$
x_{i j}{ }^{\prime}=T^{-1}\left(p_{i j}{ }^{\prime}\right)=\left\{\begin{array}{cc}
p_{i j}{ }^{\prime} X_{T} & x_{i j} \leq X_{T} \\
p_{i j}{ }^{\prime} X_{\mathrm{max}} & X_{T} \leq x_{i j} \leq X_{\mathrm{max}}
\end{array}\right.
$$

(5) According to the type (7) "min" operator, extract the effective edge of the brain tumor image: 


$$
\begin{gathered}
\left.x_{i j}{ }^{\prime \prime}=\mid x_{i j}{ }^{\prime}-\min _{Q} x_{i j}{ }^{\prime}\right\} \mid,(i, j \in Q) \\
E_{\text {edge }}=U_{i} U_{j} x_{i j}{ }^{\prime \prime}
\end{gathered}
$$

$\mathrm{Q}$ is the $3 \times 3$ window with coordinates ( $\mathrm{i}, \mathrm{j})$ as the center.

(6) Finally, get the best treatment effect of the brain tumor image edge.

According to the steps above, we run the program under the MATLAB to get the following treatment effect. Figure 5 (a) is an image of fuzzy clustering. Figure 5 (b) is the result of fuzzy enhancement edge detection on the basis of the fuzzy clustering image. By contrasting not good treatment effect of common edge detection operator in figure 2, Figure 5 (b) has not only distinctive external image edge but also clear inner edge, and even other internal structures and shapes of brain tissue can be shown. This kind of detection effect is significantly better than the traditional methods of edge detection effect, providing the high quality of post-processing slice information.

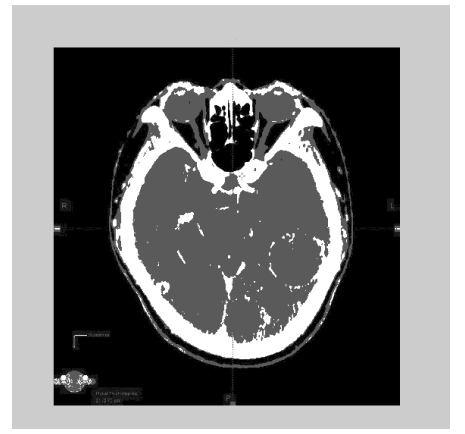

Figure 5 (a) The picture of the fuzzy clustering

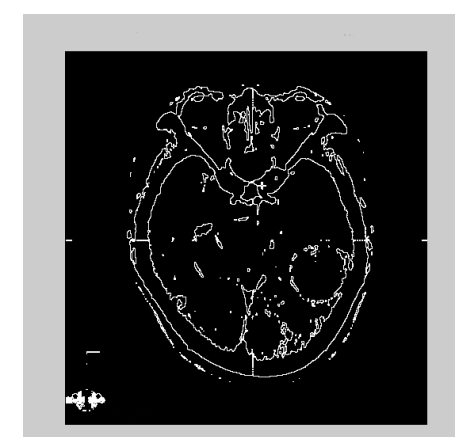

Figure 5 (b). The edges of the fuzzy enhancement test results

\section{Figure 5.The Image Comparison after Fuzzy Clustering Segmentation}

\subsection{The Post-processing Effect of the Brain Tumor Image Segmentation}

In order to illustrate the superiority of our algorithm in a better way, we provide high quality slice information for the post 3D image processing, and the specially developed language is $\mathrm{VC}++$. The design and development of the whole system is conducted through Microsoft Visual $\mathrm{c}++$ development environment called visualization toolkit VTK. Under the Windows we have achieved 3D reconstruction of the brain tumor image. According to the extracted contour of the brain tumor making 3D reconstruction, we can get the three-dimensional model of the brain tumor and the brain and implement a simple model of interaction on the 3D model. Figure 6 (a): enter into the interface of visualization system. Figure 6 (b) shows the 3D visualization processing effect of the human brain after the slice information post-processing of high quality of image segmentation. In Figure 6 (b), the brain tumor on the left ear can't be seen clearly. In order to better reflect premium quality of brain tumor biopsies in this paper, in Figure 6 (c) we extract the brain tumor out alone after reconstruction. As we can see, the edge information of the brain is obvious and shows the actual circumstance of the tumor, thus providing the scientific basis for the later diagnosis and treatment. Generally speaking, the image segmentation algorithm in this paper is very successful [9-10]. 


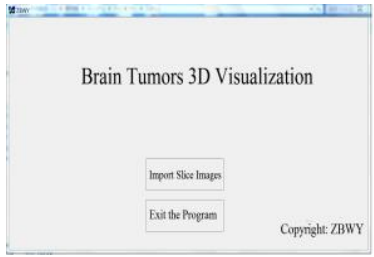

(a) The system interface diagram

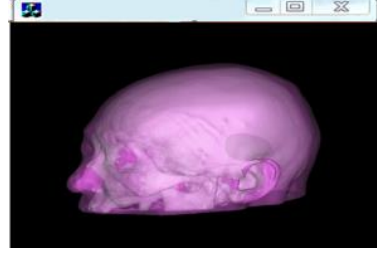

(b) Three

dimensional visualization effect

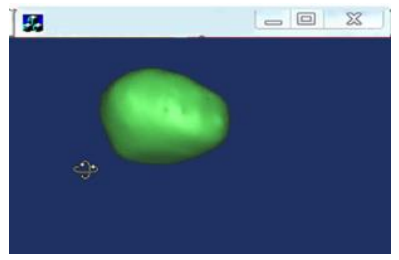

(c) Brain tumor separate extraction effect

Figure 6. Brain Tumor Image 3 d Reconstruction

\section{Conclusions}

The brain tumor image processing technologies, especially the brain tumor research and implementation of $3 \mathrm{D}$ visualization, are what matters most in helping the three dimensional conformal radiation therapies (3D-CRT) develop into the three dimensional conformal intensity modulated radiation therapy (IMRT). Moreover, it is an important approach to achieving the requirements of "precise positioning", "precision design" and "precise treatment". The brain tumor medical image segmentation technology is the key to the brain tumor medical image processing. This paper analyzes the mainstreams of brain tumor medical image segmentation methods, takes into consideration the characteristics of brain tumor image processing and grasps the development trend of the image segmentation technology. We introduce the fuzzy C-means clustering analysis method in the brain image processing, and conduct corresponding experiments where the good results have been achieved. In order to overcome the shortcomings of the traditional edge detection algorithm, we put forward the edge detection algorithm of fuzzy enhancement, which has obtained good brain tumor edge images. All the ground work has provided good slice information for the brain tumor visual image processing platform, achieved the good brain tumor image with three dimensional visualization functions and provided an important basis for medical diagnosis. We believe that our method will have a great impact on making the brain tumor medical diagnosis more scientific and accurate and it will ensure a good future in clinical application, education and medical research.

\section{Acknowledgements}

This project was sponsored in part by the National Natural Science Foundation of China (No.61302159).

\section{References}

\subsection{Journal Article}

[1] F. Leblond, S. C. Davis and P. A. Valdés, "Pre-clinical whole-body fluorescence imaging: Review of instruments, methods and applications [J]", Journal of Photochemistry and Photobiology B: Biology, vol. 98, no. 1, (2010), pp. 77-94.

[3] A. Hedström, "Automatic Segmentation of Skeleton in Whole-Body MR Images [J]", (2013).

[4] H. Pournader, M. Firouzmand and S. Ayat, "Second International Conference on Digital Image Processing [J]", (2010).

[5] J. Langner, "Event-driven motion compensation in positron emission tomography: development of a clinically applicable method [D]", University of Technology, (2008).

[6] R. P. Sørlie, "Automatic segmentation of liver tumors from MRI images [J]", Department of Physics University of Oslo, 31ST AUGUST, (2005).

[8] J. Wu, H. Xiong and C. Liu, "A Generalization of Distance Functions for Fuzzy-Means Clustering With Centroids of Arithmetic Means [J]", Fuzzy Systems, IEEE Transactions on, vol. 20, no. 3, (2012), pp. 557-571. 
[9] H. Chenini, J. P. Dérutin and R. Aufrère, "Parallel embedded processor architecture for FPGA-based image processing using parallel software skeletons [J]", EURASIP Journal on Advances in Signal Processing, 2013, vol. 1, (2013), p. 153.

\subsection{Book}

[2] "Modern computational intelligence methods for the interpretation of medical images [M]", Springer, (2008).

[7] S. Šaltenis, "Indexing the positions of continuously moving objects [M]", Springer US, (2008).

[10] S. Diehl, "Software visualization: visualizing the structure, behaviour, and evolution of software [M]", Springer, (2007).

\section{Author}

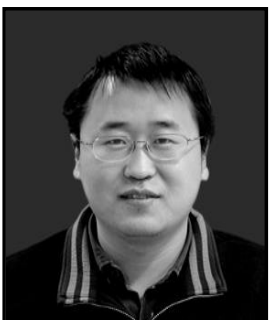

Jianning Han, School of Information and Communication Engineering, North University of China, Taiyuan Shanxi China, 
International Journal of Signal Processing, Image Processing and Pattern Recognition Vol.8, No.6 (2015) 